\title{
Discordance of the estrogen receptor and HER-2/neu in breast cancer from primary lesion to first and second metastatic site
}

This article was published in the following Dove Press journal:

Breast Cancer - Targets and Therapy

2 August 2017

Number of times this article has been viewed

\author{
Elyse E Lower' \\ Shagufta Khan ${ }^{2}$ \\ Diane Kennedy' \\ Robert P Baughman' \\ 'Department of Medicine, \\ 2Department of Pathology, University \\ of Cincinnati Medical Center, \\ Cincinnati, OH, USA
}

Background: Hormone receptor and HER-2/neu discordance between the primary lesion and first metastasis has been reported. This study was performed to determine further biomarker discordance rates between the first and subsequent metastatic breast cancer lesions.

Methods: We performed a retrospective review of paired biomarkers from primary breast cancers compared to first reported and subsequent metastases from 103 patients with breast cancer. The estrogen receptor (ER), progesterone receptor (PR), and HER-2/neu status were reported at all three time points. In addition, hormone, cytotoxic, and targeted treatments were recorded for primary and metastatic disease, and survival was determined.

Results: Between the primary and first metastases, discordance rates for ER, PR, and HER-2/neu were $15.8 \%, 33.7 \%$, and $14.3 \%$, respectively. There was discordance between the first and second metastases for the ER receptor in 18.8\%, PR receptor in 19.8\%, and HER-2/ neu in $10.7 \%$. Overall, there was discordance between the primary tumor and either the first or second metastases for ER in $27.7 \%$, PR receptor in 40.7\%, and HER-2/neu in $19.6 \%$ of cases. Discordance of either ER or PR affected survival, with worse survival experienced by those patients with all three hormone receptors remaining negative, and intermediate survival reported for those with discordant tumors (ER $\chi^{2}=14.27, p=0.0008$; PR $\chi^{2}=11.31$, $p=0.0035$ ). There was no difference in survival for patients whose HER-2/neu tumors were discordant.

Conclusion: This study demonstrated that continued metastatic disease evolution may be associated with different tumor biology and that studies of metastatic lesions appear warranted, especially if targeted therapy is an option.

Keywords: breast cancer, estrogen receptor, progesterone receptor, survival, HER-2/neu, tumor discordance

\section{Background}

Patients with hormone-dependent metastatic breast cancer often experience a lengthy disease process that can extend for years, includes multiple sites of metastasis, and requires multiple systemic treatments. Documented discordance between primary and metastatic breast cancer is reported for both hormone receptors and HER-2/ neu, with the rates of discordance ranging between $6 \%$ and $48 \% .^{1-4}$ Re-biopsy of subsequent metastasis may be important if biomarkers continue to change; however, data are lacking with regard to the possible discordance between first and subsequent metastasis, as it has not been systematically studied. Traditionally, targeted systemic treatment for early-stage disease as well as metastatic breast cancer has been based on the biomarkers identified on primary lesions. However, today, with a growing body
100I Holmes Building, 200 Albert Sabin

Way, Cincinnati, $\mathrm{OH} 45267$, USA

Email lowere@ucmail.uc.edu 
of data suggesting that discordance occurs both for hormone receptors and HER-2/neu, treatment recommendations may change based upon the prevalence of hormone receptors and HER-2/neu in metastatic disease. ${ }^{5}$

If treatment recommendations are based upon metastatic tumor analysis, retrospective studies suggest that targeted therapy changes could be needed in $20 \%$ of patients.

Although the etiology of primary metastatic discordance can include tumor sampling, processing, and analysis as well as intrinsic tumor heterogeneity, ${ }^{5}$ the actual cause of discordance can be quite elusive. To date, discordance between estrogen receptors (ER) and HER-2/neu has analyzed primary and metastatic tissue from patients with breast cancer. Little data exist regarding biomarker changes during the evolution of metastatic breast cancer. The purpose of this study is to evaluate ER and HER-2/neu biomarker changes between the primary lesion, first metastasis, and subsequent metastatic disease. In addition, the study focused on which patients may be at higher risk for changes in receptor status with subsequent sites for metastasis.

\section{Methods}

Using an established database, we conducted a retrospective chart review of the reported ER and HER-2/neu status in paired primary and subsequent metastatic invasive breast cancers between 2005 and 2014. Pathology reports were reviewed to document histology, ER and progesterone receptor (PR), and HER-2/neu status from paired samples for primary tumors and first and subsequent metastases. Not all three biomarkers were available for all reports. For all patients, demographic information was available including patient age, initial tumor stage, as well as dates of primary, first metastasis, and subsequent metastatic diagnoses. ER and PR analysis was performed using the standard immunohistochemistry of the respective pathology department. HER-2/neu analysis was performed using either standard immunohistochemistry (IHC) or fluorescent in situ hybridization (FISH). Because the reporting of hormone receptor and HER-2/neu status had changed over time, the biomarker interpretation was considered positive or negative based on the standard criteria at the time of the evaluation. The protocol was reviewed by the University of Cincinnati Institutional Review Board and we have received a waiver for obtaining patient consent to review their medical data. Patient confidentiality was maintained throughout the study as each patient was given a unique identifier and the information was stored in a password-protected file. This study was approved by the University of Cincinnati Institutional Review Board.
Comparisons were made between groups using chi-square analysis, and the overall survival from the time of initial diagnosis to the first metastasis and subsequent metastasis were calculated using Kaplan-Meier analysis. Using chisquare analysis, rates of concordance and discordance were compared between the primary lesion and the first metastasis as well as subsequent metastasis and the frequency of discordance between the different tissues.

\section{Results}

Hormone receptors and/or HER-2/neu results were available from a total of 103 patients with metastatic breast cancer. Patient demographics are summarized in Table 1, which characterizes age at diagnosis, stage at diagnosis, and median time to the first metastasis as well as median time between the first and second metastases.

Table 2 summarizes the receptor status of the primary lesion and first and second metastases when available. Not all patients had adequate stains performed for all three tumors. Patients with concordant tumors had either negative receptors for the primary lesion as well as the first and second

Table I Demographics of patients studied

\begin{tabular}{ll}
\hline Feature & \\
\hline Patients studied, $\mathrm{n}$ & 103 \\
Age, years & $48(26-80)^{*}$ \\
Time until first metastases, days & $1225(7-8 \mathrm{I} I 5)^{*}$ \\
Time between first and second metastases, days & $581(23-4769)^{*}$ \\
Tumor stage at diagnosis & \\
One & 27 \\
Two & 31 \\
Three & 5 \\
\hline
\end{tabular}

Note: *Median (range).

Table 2 Discordance between paired primary lesion and first and second metastases

\begin{tabular}{llll}
\hline Primary/first/second metastases & ER & PR & HER-2 \\
\hline Neg/neg/neg & 23 & 33 & 37 \\
Neg/neg/pos & 4 & 3 & 3 \\
Neg/pos/neg & 1 & 3 & 2 \\
Neg/pos/pos & 2 & 6 & 2 \\
Pos/neg/neg & 7 & 12 & 3 \\
Pos/neg/pos & 6 & 8 & 1 \\
Pos/pos/neg & 8 & 3 & 0 \\
Pos/pos/pos & 50 & 18 & 8 \\
Total cases studied & 101 & 86 & 56 \\
Discordance primary and first & $15.8 \%$ & $33.7 \%$ & $14.3 \%$ \\
Discordance first vs second & $18.8 \%$ & $19.8 \%$ & $10.7 \%$ \\
Discordance primary second & $20.8 \%$ & $30.2 \%$ & $14.3 \%$ \\
Overall discordance & $27.7 \%$ & $40.7 \%$ & $19.6 \%$ \\
\hline Ab & &
\end{tabular}

Abbreviations: ER, estrogen receptor; Neg, negative; Pos, positive; PR, progesterone receptor. 
metastases (neg/neg/neg) or positive staining for receptors in the primary lesion as well as the first and second metastases (pos/pos/pos). When we compared the primary tumor to the first metastases, there was concordance between the ER, PR, and HER-2/neu analysis in the majority of cases. However, there was discordance for ER in $15.8 \%$, PR in $33.7 \%$, and HER-2/neu in $14.3 \%$ of cases.

Furthermore, we calculated the rate of discordance between the paired first and second metastases. Again, the majority of tumors were concordant. There was discordance between the paired first and second metastasis for the ER receptor in $18.8 \%$ of cases, for the PR receptor in $19.8 \%$ of cases, and for HER-2/neu in $10.7 \%$ of cases. There were similar discordance rates between the paired primary lesion and second metastasis: ER receptor in $20.8 \%$ of cases, PR receptor in $30.2 \%$, and for HER-2/neu in $14.3 \%$ of cases.

Overall, there was discordance between the primary tumor and either the first or second metastases in a significant number of cases. For the ER receptor, it was in $27.7 \%$ of cases, for the PR receptor it was in $40.7 \%$ of cases, and for HER-2/neu it was in $19.6 \%$ of cases. This would include cases where the primary tumor was positive, the first metastasis was negative, and the second metastases remained positive or vice versa.

We then calculated the survival of patients from initial diagnosis for three scenarios: primary tumor negative and both the first and second metastases were also negative; primary tumor positive and both the first and second metastases were positive; and discordance between the primary lesion and either the first and/or the second metastases. Figure 1 shows the survival curve based on the ER status. There was a significant difference in survival, with the best survival noted in patients with biomarkers positive at all three time points. Worse survival was witnessed for patients with all three tumors negative for biomarkers, and intermediate survival for those with discordant tumors (chi-square $=14.27$, $p=0.0008$ ). Figure 2 shows the survival curve based on the PR status. Again, there was a significant difference between the survival curves (chi-square $=11.31, p=0.0035$ ). In this case, patients with tumors negative for PR at all three time points experienced a worse survival probability, whereas survival was similar for the other two situations. In contrast to hormone receptor discordance, there was no difference in survival for the three HER-2/neu concordant or discordant groups (Figure 3, chi-square $=4.66, p=0.972$ ).

\section{Discussion}

Because most targeted treatment decisions are recommended on the basis of biomarkers for hormone receptors and HER-2/neu,

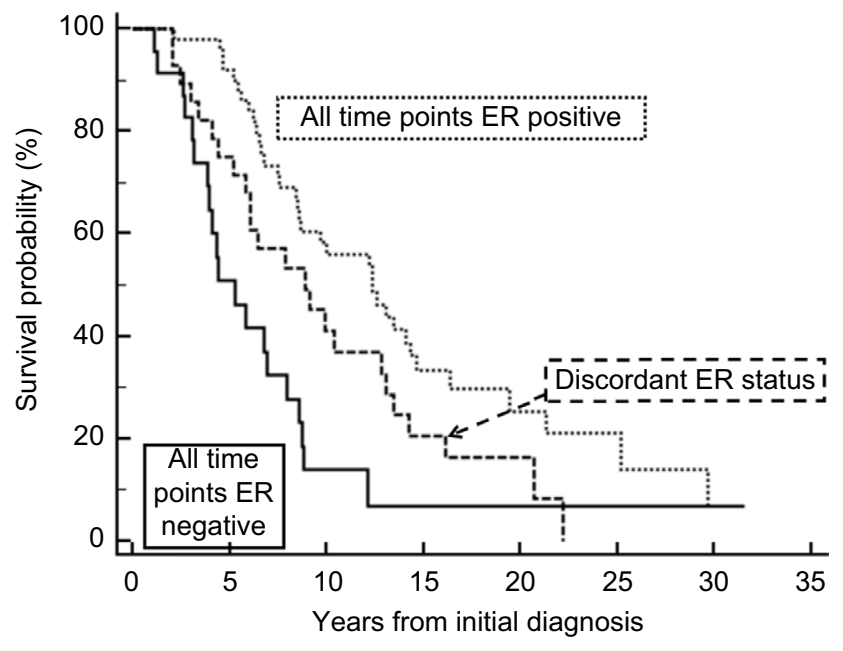

Figure I Survival curve based on the estrogen receptor (ER) status. There was a significant difference in survival, with the best survival noted in patients with biomarkers positive at all three time points. Worse survival was witnessed for those patients with all three parameters negative, and intermediate survival for those with discordant tumors (chi-square $=14.27, p=0.0008$ ).

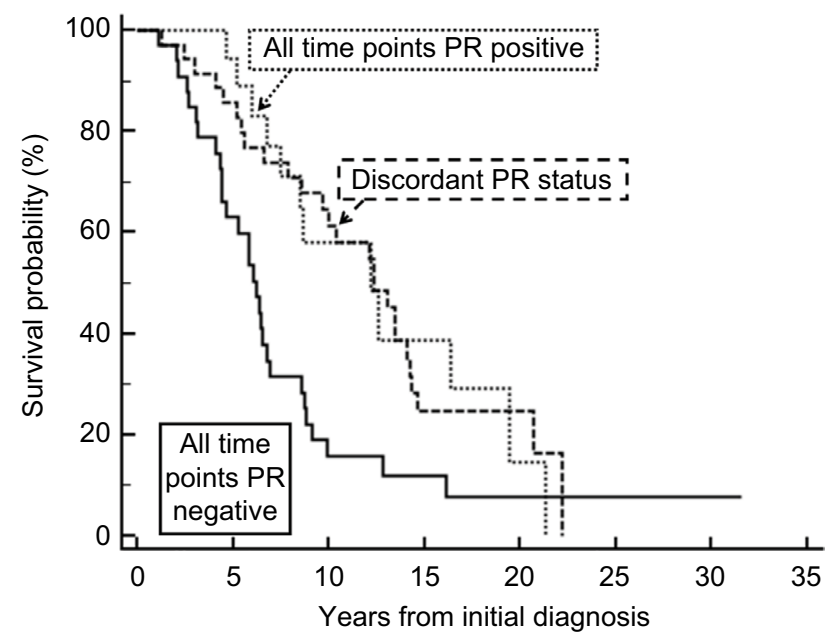

Figure 2 Survival curve based on the progesterone receptor (PR) status. Again there was a significant difference between the survival curves (chi-square $=|1| .31$, $p=0.0035$ ). In this case, patients with tumors negative for PR at all three time points experienced a worse survival probability, whereas survival was similar for the other two situations.

a change in biomarkers could have a dramatic impact on systemic treatment recommendations. ${ }^{5-7}$ Certainly, patients whose primary hormone receptor or HER-2/neu tumor biomarkers are negative and subsequent tumors are rendered positive provide great opportunity for additional targeted systemic management with hormone manipulation or HER-2/neu-targeted treatments. We and others have previously shown a substantial discordance rate between primary tumor and metastatic disease, with studies reporting hormone receptor discordance rates between $30 \%$ and $40 \%$ and HER-2/neu discordance rates between $10 \%$ and $30 \%{ }^{1,3,4,8-10}$ Although the American College of Pathologists recommends biomarker analysis on primary and metastatic 


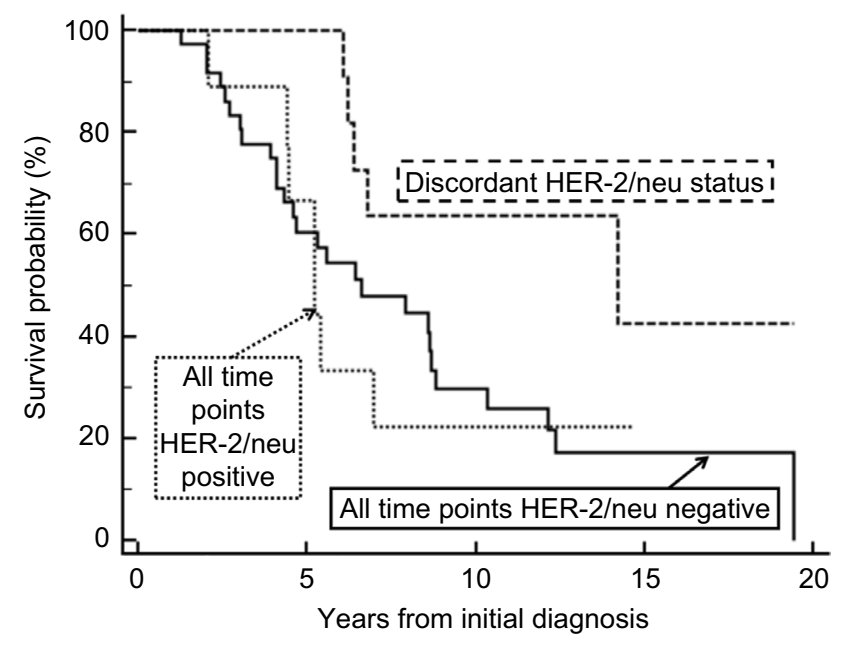

Figure 3 Demonstrates the survival curves for the three scenarios based on HER-2/ neu status. In contrast to hormone receptor discordance, there was no difference in survival for the three HER-2/neu concordant or discordant groups (chi-square=4.66, $p=0.972$ ).

breast cancers, ${ }^{13}$ to the authors' knowledge this is the first report which analyzes the continued biomarker discordance of primary breast cancer with paired specimens of first and subsequent metastases. Our current study examined the markers on paired samples from subsequent sites of metastasis. We demonstrated overall discordance rates between $20 \%$ and $40 \%$. In particular, we found discordance between the first and second metastases of approximately $20 \%$ for ER and PR receptors and $11 \%$ for HER-2/neu.

The lack of standardized pre-analytic and analytic variables previously may have accounted for some of these discrepancies between primary and metastatic lesions, as immunohistochemical analysis is dependent upon the timely placement of the specimen in fixative as well as specimen handling and the total fixation time. Adherence to the American Society of Clinical Oncology/College of American Pathologists (ASCO/CAP) guidelines should improve antigen retrieval and provide for more accurate IHC analysis of breast cancer specimens. ${ }^{11,12}$ Additionally, the interpretation of hormone receptor positivity has changed throughout the years. ${ }^{12}$ The current cutoff point for receptor positivity can be very low ( $>1 \%$ ); therefore, minor differences in antibody sensitivity can alter hormone receptor status. Current ASCO CAP guidelines require the hormone receptor status to be reported as positive or negative based upon a semiquantitative score of both the number of invasive tumor cells staining positively and the intensity of the stain. This system should better predict responses to hormone therapy and be easier and more cost-effective. Identifying the HER-2/neu-driven tumor has been vexing. Current guidelines define a breast tumor as HER-2/neu positive using either IHC or FISH. ${ }^{11}$ Recent changes in FISH positivity have modified the cutoff point to take into account both the HER-2/neu signal to chromosome 17 ratio as well as the gene copy number. Many systems still use HER-2/neu analysis by immunohistochemistry, wherein a score of $3+$ is considered positive and $2+$ score necessitates subsequent reflex testing. ${ }^{13}$ Currently, approximately $15-20 \%$ of all invasive ductal cancers will be considered HER-2/neu driven, using either a $3+$ IHC score or FISH amplification. Most studies suggest that HER-2/neu concordance rates between IHC and FISH are approximately 90-96\%.. ${ }^{11,13}$ Unfortunately, inaccurate interpretation of HER-2/neu can result in false negatives which could deny patients HER-2/ neu-targeted treatment, which can improve response rates and overall survival.

Etiology of primary and metastatic discordance can also involve tumor sampling as well as intrinsic tumor heterogeneity. Heterogeneous tumor clones with different hormone receptor and HER-2/neu biomarkers can occur as the result of host environmental changes or treatment consequences. ${ }^{14}$ In particular, tumors from patients who previously received adjuvant hormone or HER-2/neu-targeted treatment may develop receptor downregulation or resistance. Discordance between primary and metastatic breast cancer has been suggested for many years; however, most studies were retrospective, with only a few prospective studies now reporting biomarker discordance rates. ${ }^{8,9}$

Only a few studies discuss the clinical importance of such discordance. ${ }^{15}$ The chance of discordance between the primary metastatic tumors for both hormone receptors approximates 20-30\%. We previously reported that ER discordance was associated with changes in survival. ${ }^{4}$ Other investigators have also noted discordance affecting survival. Better survival was demonstrated in patients with either primary or metastatic disease ER positivity compared to those with ER-negative disease. ${ }^{14,16,17}$ However, survival advantage was not significant in all studies ${ }^{18}$ which may reflect on study size or duration of follow-up.

Many patients with metastatic breast cancer witness prolonged survival which can be associated with the development of multiple sites of metastasis with differing biology. This study reveals the impact of tumor discordance which could occur during the evolution of metastasis. We studied hormone receptor and HER-2/neu discordance in primary, first, and second metastatic disease, and we noted the best survival was experienced by patients whose tumors remained ER positive at all three time points whereas significantly worse survival was found in those patients whose tumors remained ER negative at all three time points. Patients with 
ER discordance between either the first or second metastases demonstrated intermediate survival. The finding was similar for the PR as well.

In our current study, discordance between HER-2/neu receptors failed to impact survival. This result is similar to findings from previous studies which compared primary and first metastatic disease sites. ${ }^{3,14}$ Although most studies do not report discordance rates for HER-2/neu-negative primary and metastatic tumors, some studies have noted a worse prognosis for those patients whose tumors lose HER-2/neu positivity over time. ${ }^{17-20}$ Additionally, there are cases in which a patient's primary tumor was HER-2/neu negative but the metastasis was considered HER-2/neu positive. ${ }^{21}$ In only approximately $7 \%$ of 800 cases was discordance reported for primary HER-2/neu negativity seen to change to metastatic HER-2/ neu positivity. ${ }^{19}$ In our current study, seven of 44 patients (16\%) with HER-2/neu negative primary tumors had either their first or second metastases recorded as HER-2/neu positive. Although HER-2/neu-positive breast cancer is associated with aggressiveness and impaired survival, treatment with HER-2/neu-targeted therapies including trastuzumab is quite effective in neutralizing the impact of HER-2/neu on survival.

Large studies report primary HER-2/neu-positive tumors with discordant metastatic disease in $24-64 \%$ of patients. ${ }^{16,17,19}$ However, bias may cloud these retrospective studies due to a tendency to repeat HER-2/neu testing only in patients considered likely to have discordant tumors. ${ }^{21}$ In our study of unselected cases, only four of $12(33 \%)$ patients whose primary tumors were HER-2/neu positive experienced HER-2/neu negativity in either the first or second metastases.

As with ER discordance, the etiology of HER-2/neu change is probably multifactorial and includes pre-analytical as well as analytical factors. ${ }^{5,22}$ Tumor heterogeneity makes tissue sampling very problematic. Small case series suggest that HER-2/neu discordance occurs in some patients who have received neoadjuvant trastuzumab for HER-2/neupositive primary cancers.

Current systemic treatment recommendations are based on primary tumor assessment, prior adjuvant treatments, time to relapse, and sites of metastasis. A wide range of systemic treatments are available for patients whose tumors are hormone receptor positive and/or HER-2/neu positive. Fifty percent improvement in survival has been identified in patients who have received adjuvant HER-2/neu-targeted trastuzumab. ${ }^{23}$ Obviously, a false-negative biomarker interpretation could deny a patient a potentially life-saving treatment. Likewise, patients whose tumors are not truly hormone receptor positive or HER-2/neu driven should be spared the toxicity and cost of inappropriate therapy. Newer guidelines suggest serial HER-2/neu evaluation to more precisely offer anti-HER-2/neu treatment in the metastatic setting.

This study confirms the previously reported discordance rates of approximately $25 \%$ between primary and metastatic breast cancer for hormone receptors and HER-2/neu. This discordance was noted for both the primary tumor and first metastasis as well as subsequent metastatic disease. Patients whose primary lesions are ER negative and whose metastatic lesion is ER positive could benefit from adjuvant hormone therapy. Likewise, changes in HER-2/neu can effect treatments. This study points out that one should consider reevaluating tumor biomarkers whenever a new metastatic lesion occurs. For example, it may help direct therapy for a patient whose previous tumor was ER positive but is no longer responsive to hormone therapy. Further studies will be needed to determine the potential role of routine repeat testing of tumor biomarker status for new metastatic lesions.

\section{Conclusion}

This study demonstrated that continued metastatic disease evolution may be associated with different tumor biology. Changes in tumor markers between first and second metastases can occur in up to $20 \%$ of cases. Therefore, studies of additional metastatic lesions appear warranted, especially if targeted therapy is an option.

\section{Author contributions}

EEL designed the study. DK was responsible for data collection and entry. EEL and RPB performed data analysis and manuscript preparation. All authors contributed toward data analysis, drafting and critically revising the paper, gave final approval of the version to be published, and agree to be accountable for all aspects of the work.

\section{Disclosure}

The authors report no conflicts of interest in this work.

\section{References}

1. Jensen JD, Knoop A, Ewertz M, Laenkholm AV. ER, HER2, and TOP2A expression in primary tumor, synchronous axillary nodes, and asynchronous metastases in breast cancer. Breast Cancer Res Treat. 2012;132(2):511-521

2. Broom RJ, Tang PA, Simmons C, et al. Changes in estrogen receptor, progesterone receptor and Her-2/neu status with time: discordance rates between primary and metastatic breast cancer. Anticancer Res. 2009;29(5):1557-1562.

3. Lower EE, Glass E, Blau R, Harman S. HER-2/neu expression in primary and metastatic breast cancer. Breast Cancer Res Treat. 2009;113(2):301-306.

4. Lower EE, Glass EL, Bradley DA, Blau R, Heffelfinger S. Impact of metastatic estrogen receptor and progesterone receptor status on survival. Breast Cancer Res Treat. 2005;90(1):65-70. 
5. Lower EE, Khan S. Biomarker discordance: why it occurs and why it is important. Cancer Biomark. 2012-2013;12(6):219-230.

6. Van Poznak C, Somerfield MR, Bast RC, et al. Use of biomarkers to guide decisions on systemic therapy for women with metastatic breast cancer: American Society of Clinical Oncology Clinical Practice Guideline. J Clin Oncol. 2015;33(24):2695-2704.

7. Rocca A, Farolfi A, Maltoni R, et al. Efficacy of endocrine therapy in relation to progesterone receptor and $\mathrm{Ki} 67$ expression in advanced breast cancer. Breast Cancer Res Treat. 2015;152(1):57-65.

8. Kulka J, Székely B, Lukács LV, et al. Comparison of predictive immunohistochemical marker expression of primary breast cancer and paired distant metastasis using surgical material: a practice-based study. $J$ Histochem Cytochem. 2016;64(4):256-267.

9. Li MH, Hou CL, Wang C, Sun AJ. HER-2, ER, PR status concordance in primary breast cancer and corresponding metastatic lesion in lymph node in Chinese women. Pathol Res Pract. 2016;212(4):252-257.

10. Domanski AM, Monsef N, Domanski HA, Grabau D, Fernö M. Comparison of the oestrogen and progesterone receptor status in primary breast carcinomas as evaluated by immunohistochemistry and immunocytochemistry: a consecutive series of 267 patients. Cytopathology. 2013;24(1):21-25.

11. Wolff AC, Hammond ME, Hicks DG, et al. Recommendations for human epidermal growth factor receptor 2 testing in breast cancer: American Society of Clinical Oncology/College of American Pathologists clinical practice guideline update. J Clin Oncol. 2013;31(31):3997-4013.

12. Hammond ME, Hayes DF, Dowsett M, et al. American Society of Clinical Oncology/College Of American Pathologists guideline recommendations for immunohistochemical testing of estrogen and progesterone receptors in breast cancer. J Clin Oncol. 2010;28(16):2784-2795.

13. Singh K, Tantravahi U, Lomme MM, Pasquariello T, Steinhoff M, Sung CJ. Updated 2013 College of American Pathologists/American Society of Clinical Oncology (CAP/ASCO) guideline recommendations for human epidermal growth factor receptor 2 (HER2) fluorescent in situ hybridization (FISH) testing increase HER2 positive and HER2 equivocal breast cancer cases; retrospective study of HER 2 FISH results of 836 invasive breast cancers. Breast Cancer Res Treat. 2016;157(3):405-411.
14. Karlsson E, Appelgren J, Solterbeck A, Bergenheim M, Alvariza V, Bergh J. Breast cancer during follow-up and progression - A population based cohort on new cancers and changed biology. Eur J Cancer. 2014;50(17): 2916-2924.

15. Ibrahim T, Farolfi A, Scarpi E, et al. Hormonal receptor, human epidermal growth factor receptor-2, and Ki67 discordance between primary breast cancer and paired metastases: clinical impact. Oncology. 2013;84(3): $150-157$.

16. Ba JL, Liu CG, Jin F. Alterations in hormonal receptor expression and HER2 status between primary breast tumors and paired nodal metastases: discordance rates and prognosis. Asian Pac J Cancer Prev. 2014;15(21):9233-9239.

17. Matsumoto A, Jinno H, Murata T, et al. Prognostic implications of receptor discordance between primary and recurrent breast cancer. Int J Clin Oncol. 2015;20(4):701-708.

18. Karagöz Özen DS, Ozturk MA, Aydin Ö, Turna ZH, Ilvan S, Özguroglü M. Receptor expression discrepancy between primary and metastatic breast cancer lesions. Oncol Res Treat. 2014;37(11):622-626.

19. Niikura N, Liu J, Hayashi N, et al. Loss of human epidermal growth factor receptor 2 (HER2) expression in metastatic sites of HER2-overexpressing primary breast tumors. J Clin Oncol. 2012;30(6):593-599.

20. Yang YF, Liao YY, Yang M, Peng NF, Xie SR, Xie YF. Discordances in ER, PR and HER2 receptors between primary and recurrent/metastatic lesions and their impact on survival in breast cancer patients. Med Oncol. 2014;31(10):214.

21. Turner NH, Di Leo A. HER2 discordance between primary and metastatic breast cancer: assessing the clinical impact. Cancer Treat Rev. 2013;39(8):947-957.

22. Asogan AB, Hong GS, Arni Prabhakaran SK. Concordance between core needle biopsy and surgical specimen for ER, PgR and Her2neu receptor status in breast cancer and study of difference in reliability between the two groups with the change in ASCO/CAP guidelines. Singapore Med J. 2016;10.

23. Viani GA, Afonso SL, Stefano EJ, De Fendi LI, Soares FV. Adjuvant trastuzumab in the treatment of her-2-positive early breast cancer: a meta-analysis of published randomized trials. BMC Cancer. 2007;7:153.
Breast Cancer - Targets and Therapy

\section{Publish your work in this journal}

Breast Cancer - Targets and Therapy is an international, peerreviewed open access journal focusing on breast cancer research, identification of therapeutic targets and the optimal use of preventative and integrated treatment interventions to achieve improved outcomes, enhanced survival and quality of life for the cancer patient.

\section{Dovepress}

The manuscript management system is completely online and includes a very quick and fair peer-review system, which is all easy to use. Visit http://www.dovepress.com/testimonials.php to read real quotes from published authors. 